\title{
Primary Renal Lymphoma (Low-grade B cell Follicular Lymphoma) Presenting as Pyrexia of Unknown Origin
}

\author{
Naman Sahu, Banwari Lal, Umesh Kansra, Baljinder Kaur \\ Departments of Internal Medicine, Metro Heart Institute with Multispeciality, Sector 16A, Faridabad- 8103559894, Haryana, India.
}

\section{Corresponding Author: \\ Dr Naman Sahu \\ Email: namansmoon@gmail.com}

This is an Open Access article distributed under the terms of the Creative Commons Attribution License (creativecommons.org/ licenses/by/3.0).

Received Accepted

Published

October 19, 2020

August 13, 2021

October 5, 2021

\begin{abstract}
Background: Primary renal lymphoma (PRL) is a disease of genitourinary tract which is a rare entity; even its existence has been debated because of absence of lymphatic tissue within renal parenchyma per se. Case Report: We present the case of 67-yr old male who was evaluated for pyrexia of unknown origin and refractory oral ulcer. His NCCT whole abdomen revealed retroperitoneal mass arising from mid and lower pole of right kidney. Histopathology and immunohistochemistry of ultrasound guided renal biopsy revealed low grade B cell follicular non-Hodgkin lymphoma, a rare entity of primary renal non-Hodgkin lymphoma. Conclusion: Primary renal lymphoma (PRL) should be kept as differential diagnosis while investigating renal mass.
\end{abstract}

Keywords: Biopsy, Fever, Kidney, Non-Hodgkin Lymphoma, Oral Ulcer.

\section{Introduction}

Primary renal non-Hodgkin's lymphoma (NHL) is a rare disease, as the kidney is an extra-nodal organ with no lymphatic tissue [1]. Therefore, its existence has been continuously questioned however, in recent years, reports of primary renal lymphoma(PRL) cases have confirmed the presence of the disease. PRL has been shown to account for $0.7 \%$ of all extra-nodal lymphomas in North America and $0.1 \%$ of all malignant lymphomas in Japan [2]. No more than 70 cases of PRL have been reported in the literature and the majority are of NHL large B cell type [3]. The precise cause of PRL remains unknown. It has been suggested that PRL originates from the renal capsule and infiltrates the renal parenchyma. Another theory is that chronic inflammatory conditions of the kidney attract the infiltration of lymphoid cells, and eventually evolve into lymphoma [4,5]. PRL lacks clear clinical manifestations and appears similar to that of renal cell carcinoma (RCC), renal abscess and other renal metastasis. Hence, a kidney biopsy remains the gold standard for the diagnosis of primary renal lymphoma [6].

In 2020 Taneja A et al. in a population base analysis using the SEER program (1973-2015) found a total of $599(0.17 \%$ of all NHL) cases of PRL. Majority (89.6\%) of PRL was B cell type NHL. The most common was diffuse large B cell lymphoma (DLBCL) (45.7\%) followed by extranodal marginal zone lymphomas (MZL) [14.4\%] and follicular lymphomas (FL) [11.4\%] and only $1.2 \%$ of the PRL were of the T-cell type [5]. Our case is unique because of its atypical presentation. In addition, a low-grade B cell follicular nonHodgkins lymphoma type is extremely rare.

\section{Case Report}

We hereby report case of 67 year-old male who was apparently alright 2 months back when he developed an oral ulcer and intermittent fever for which he received treatment locally but had no relief rather his symptoms were aggravated. He subsequently visited our center for dermatology 
consultation and was being treated as Stevens Johnson syndrome but his symptoms persisted and further developed high grade fever. He was referred to medicine department in view of non-healing oral ulcer and pyrexia of unknown origin for further evaluation and management. He had no history of weight loss, pain abdomen, hematuria or loss of appetite. On examination, he was moderately build and nourished, febrile with absence of peripheral lymphadenopathy and hepatosplenomegaly. Oral cavity was erythematous, ulcerated with pus foci, and bleeding from lips [Fig.1].

His routine blood investigations, complete blood picture and liver function test were normal. Renal functions were deranged (serum urea 54 $\mathrm{mg} / \mathrm{dL}$, serum creatinine $1.8 \mathrm{mg} / \mathrm{dL}$ ), Escherichia coli was isolated from oral pus culture; urine routine/microscopy revealed no proteins, no RBC; peripheral blood smear was normal. Viral markers (HIV, HCV, HBsAg) were non-reactive. Chest $\mathrm{X}$-ray was normal. Ultrasound (USG) abdomen revealed heterogeneously hypo-echoic lesion to lower pole region of right kidney with perinephric involvement and extending up to psoas with moderate right sided hydroureteronephrosis. NCCT whole abdomen showed [Fig.2,3] a large homogenous retroperitoneal mass arising from mid and lower pole of right kidney causing compression of inferior venae cava (IVC) with possible invasion of bilateral renal veins with well-defined soft tissue attenuation lesion in left hemi-pelvis abutting left external iliac vessels, and a homogenous soft tissue attenuation lesion in visualized posterior mediastinum partially encasing the descending thoracic aorta, suggestive of neoplastic etiology suspected cause being renal cell carcinoma and metastasis.

USG guided trucut biopsy taken from right renal lower pole and left iliac lymph node was suggestive of NHL. Histopathological examination and immunohistochemistry revealed positive CD20, CD21, CD10, BCL2; BCL 6 was

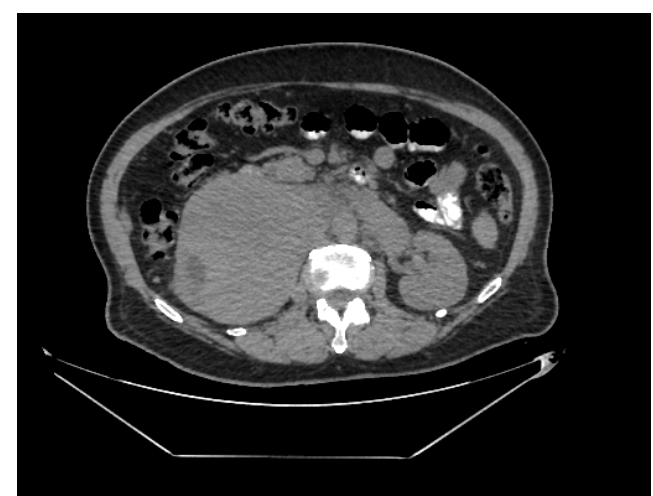

Fig.1: NCCT whole-abdomen (axial view): a large homogenous retroperitoneal mass arising from mid and lower pole of right kidney.

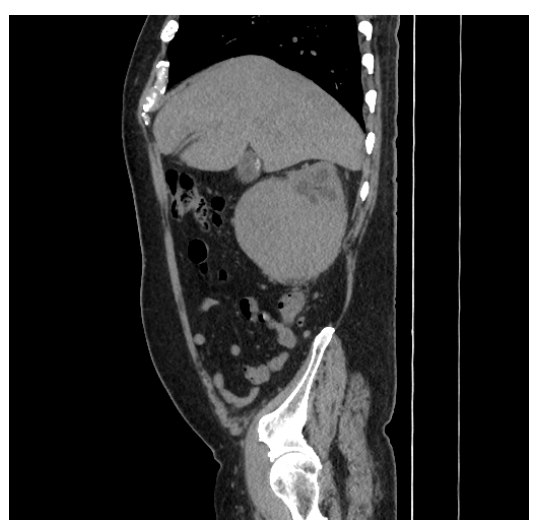

Fig.2: NCCT whole abdomen (sagittal view): a large homogenous retroperitoneal mass arising from mid and lower pole of right kidney.

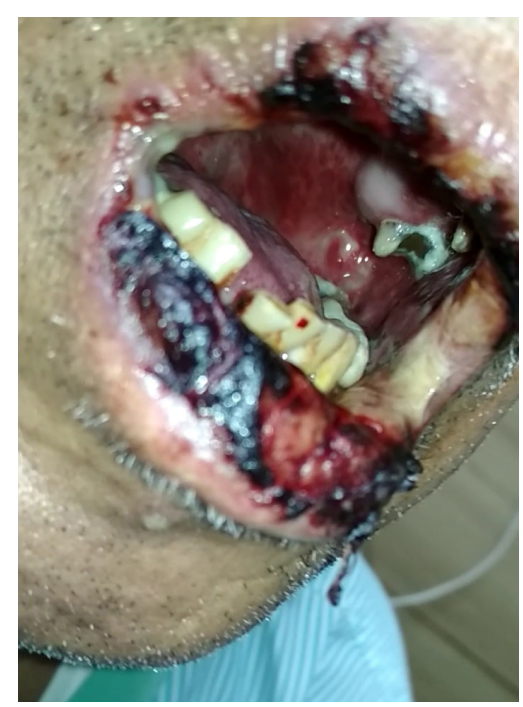

Fig.3: Oral cavity was erythematous, ulcerated with pus foci, and bleeding from lips. 
focal weak positive; Ki-67 was approx. $15 \%$; cyclin D1, CD3, CD5, CD23, CD 43 and MUM-1 were negative, and he was diagnosed as a low grade $b$ cell follicular non-Hodgkins lymphoma (NHL).

\section{Discussion}

The diagnosis of PRL includes the presence of a renal mass; no evidence of extra-renal lymphomatous involvement in the visceral organs or lymph nodes at first admission; and the absence of a leukemic blood picture together with no evidence of myelosuppression. However, a kidney biopsy remains the gold standard for the diagnosis of primary renal lymphoma [6]. Chen $\mathrm{X}$ et al. found that PRL generally appears to be bilateral in patients who are younger than 18 years old and unilateral in adult patients. Fever is the most common symptom in younger patients $(56 \%)$, while abdominal and flank pain are common (62\%) in patients aged from 18-50 years. Weight loss and gross hematuria are the most common symptoms (37\%) for patients who are older than 50 years [7]. whereas our patient had atypical features, nonhealing ulcer and pyrexia of unknown origin. PRL is considered as a systemic disease, presents with renal manifestation. However, PRL patients can present with atypical features of RCC, therefore, pre-operative percutaneous renal biopsy should be done. The sensitivity and specificity of renal biopsy are $70-92 \%$ and $100 \%$ respectively with accuracy close to $90 \%$ [8]. Poor prognosis is reported universally and median survival is less than a year [8].

Our patient had a solitary mass in the right kidney. Though lymph nodes were demonstrable in the retroperitoneum, the absence of disease elsewhere enhances the probable site of origin was renal. This case is important to the treating surgeon to be enlightened about PRL which can mimic RCC, radiologically and clinically, so that nephrectomy can be avoided with an early diagnosis. The treatment of renal lymphoma depends on the primary histological subtype. The standard R-CHOP chemotherapy may improve the morbid outcome reported so far [9].

\section{Conclusion}

Despite the rarity of its incidence, primary renal lymphoma (PRL) should be kept as differential diagnosis while investigating renal mass since its management and prognosis varies so vastly from the usual suspects like renal cell carcinoma (RCC), renal abscess and other renal metastases. While the diagnosis is mostly radiological, it is essential to confirm the same histologically to justify the initiation of chemotherapy as primary modality of treatment.

Contributors: NS, BL: manuscript writing, patient management; UK, BK: manuscript editing, patient management. BK will act as a study guarantor. All authors approved the final version of this manuscript and are responsible for all aspects of this study.

Funding: None; Competing interests: None stated.

\section{References}

1. Paganelli E, Arisi L, Ferrari ME, Olivetti G, Tedeschi F. Primary non Hodgkin's lymphoma of the kidney. Haematologica. 1989;74:301-304.

2. Aozasa K, Tsujimoto M, Sakurai M, Honda M, Yamashita $\mathrm{K}$, Hanada M, et al. Non Hodgkin's lymphomas in Osaka, Japan. Eur J Cancer Clin Oncol. 1985;21:487-492.

3. Belbaraka R, Elyoubi MB, Boutayeb S, Errihani H. Primary renal non Hodgkin lymphoma: An unusual diagnosis for a renal mass. Indian J Cancer. 2011;48:255256

4. Gellrich J, Hakenberg OW, Naumann R, Manseck A, Lossnitzer A, Wirth MP. Primary renal non Hodgkin's lymphoma a difficult differential diagnosis. Onkologie. 2002;25:273-277.

5. Taneja A, Kumar V, Chandra AB. Primary renal lymphoma: A population-based analysis using the SEER program (1973-2015). Eur J Haematol. 2020;104:390399.

6. Al Salam S, Shaaban A, Alketbi M, Haq NU, Abouchacra S. Acute kidney injury secondary to renal large B cell lymphoma: Role of early renal biopsy. Int Urol Nephrol. 2011;43:237-240.

7. Chen X, Hu D, Fang L, Chen Y, Che X, Tao J, et al. Primary renal lymphoma: A case report and literature 
review. Oncology letters. 2016;12(5):4001-4008.

8. Skarin A. Uncommon presentations of non-Hodgkin's lymphoma. Case 3, Primary renal lymphoma. J Clin Oncol. 2003;21:564-569.
9. Cyriac S, Rejiv R, Shirley S, Sagar GT. Primary renal lymphoma mimicking renal cell carcinoma. Indian $\mathrm{J}$ Urol. 2010;26:441-443. 